\title{
AVALIAÇÃO DOS PLANOS DE GERENCIAMENTO DE RESÍDUOS DE SERVIÇOS DE SAÚDE DE FARMÁCIAS COMUNITÁRIAS PRIVADAS ENTREGUES A VIGILÂNCIA SANITÁRIA DE UM MUNICÍPIO DA REGIÃO METROPOLITANA DO RECIFE
}

EVALUATION OF HEALTH SERVICES WASTE MANAGEMENT PLANS OF PRIVATE COMMUNITY PHARMACIES DELIVERED TO THE HEALTH SURVEILLANCE OF A CITY OF THE METROPOLITAN RECIFE REGION

Tássya Rebecka Neves Araújo ${ }^{1}$ Luana Layse Câmara de Almeida ${ }^{2}$ Raissa Daniel Trajano dos Santos ${ }^{3}$ Adriana Amorim de Farias Leal

Rômulo Moreira dos Santos

RESUMO: OBJETIVOS: Analisar a gestão dos resíduos sólidos declarados por farmácias comunitárias privadas através do PGRSS entregues à vigilância sanitária local. MÉTODO: Estudo de prevalência, com abordagem quantitativa, realizado nos meses de junho a dezembro de 2019, no Município de Goiana - PE, junto as farmácias comunitárias. RESULTADOS: Participaram da pesquisa 26 farmácias comunitárias, das quais todas foram declaradas de pequeno porte, um quinto produz resíduos dos tipos $A$ e $E$, sendo responsáveis pelo acondicionamento e tratamento dos mesmos, visando cada vez mais a responsabilidade quanto a saúde da população e ao meio ambiente, seguindo desta forma corretamente a legislação vigente. A incineração foi empregada como tratamento final, quando necessário, por todas as unidades de saúde. CONCLUSÃO: Apesar dos dados secundários apresentarem informações quanto ao seguimento das normas, faz-se necessário fiscalizações e investimentos na educação em saúde, objetivando a manutenção do ecossistema para as gerações futuras.

\footnotetext{
1 Graduanda do curso de Farmácia da Unifacisa.

2 Estudante do Programa de Pós-graduação em Saúde Coletiva da UFRN.

3 Farmacêutico, Mestre em Saúde Pública e Professor dos cursos de Farmácia e Nutrição da Unifacisa.
} 
Palavras chave: Farmacoepidemiologia; Resíduos de Serviços de Saúde; Serviços comunitários de farmácia.

ABSTRACT: OBJECTIVES: To analyze the management of solid waste declared by private community pharmacies through PGRSS delivered to local health surveillance. METHOD: Prevalence study, with quantitative approach, conducted from June to December 2019, in the city of Goiana - PE, with community pharmacies. RESULTS: Participated in the research 26 community pharmacies, all of which were declared small, one fifth produces waste types $A$ and $E$, being responsible for their packaging and treatment, aiming increasingly the responsibility for the health of the population and the environment, correctly following current legislation. Incineration was used as final treatment, when necessary, by all health facilities. CONCLUSION: Although the secondary data present information regarding the compliance with the norms, it is necessary to supervise and invest in health education, aiming at maintaining the ecosystem for future generations.

Keywords: Pharmacoepidemiology; Health services waste; Community pharmacy services. 


\section{INTRODUÇÃO}

É sabido que todo ser vivo reage com o ambiente a qual estão inseridos e assim produzem resíduos. O ser humano tem produzido inúmeras quantidades de resíduos e subprodutos, os quais tem um profundo significado político, sanitário e social, que futuramente poderão interferir no ciclo vital de todos os seres vivos e na poluição do meio ambiente. Diante disso, podemos definir como poluição ambiental a degradação do ambiente, que é resultado de atividades que prejudiquem a saúde, as condições sanitárias, a segurança e bem-estar da população, fauna e flora. (DERISIO, 2017).

É perceptível que o descarte inadequado dos resíduos sólidos produzem malefícios ao meio ambiente, podendo ocasionar comprometimento dos recursos naturais e consequentemente piora na qualidade de vida dos seres humanos e animais. Os resíduos de serviços de saúde (RSS) também estão inclusos neste impasse, o qual ganha cada vez mais destaque na atualidade (BRASIL, 2006).

De acordo com Costa et al., 2018, um dos problemas que também assola o meio ambiente é o descarte inadequado nos lixões a céu aberto, visto que, os resíduos são queimados prejudicando cada vez mais nosso ecossistema. Diante do estudo do realizado por ele, a necessidade da educação ambiental também é um ponto primordial para manutenção da natureza e promoção da segurança do homem.

Como citado anteriormente, o investimento em educação é necessário para conscientização, orientação, motivação e entre outras características visando cada vez mais as informações sobre os riscos e as adequações quanto ao gerenciamento dos resíduos. (Pinheiro e Silva, 2016).

Tendo em vista todo os riscos e com o propósito de minimizar os danos à saúde e ao meio ambiente, foi sancionada a Política Nacional de Resíduos Sólidos (PNRS), pela Lei 12.305, de 2 de agosto de 2010, que tem como principal objetivo a gestão e gerenciamento dos resíduos sólidos (BRASIL, 2010). 
Segundo Santos, Ruiz e Camilo (2017), no Brasil, os RSS compõem cerca de $2 \%$ da quantidade total de resíduos sólidos urbanos residenciais e comerciais. E com isso houve a necessidade da criação da RDC 306, de 7 de dezembro de 2004, que foi atualizada recentemente pela RDC 222/2018 (BRASIL, 2018). Ela consiste que todo gerador deve elaborar um Plano de Gerenciamento de Resíduos de Serviço de Saúde (PGRSS), baseado nas características e classificação dos resíduos gerados, estabelecendo as diretrizes de manejo do lixo produzido.

Além disso, a Resolução CONAMA N ${ }^{\circ} 385$ de 2005 também trata do gerenciamento dos RSS em todas as suas etapas, no entanto, o foco da mesma é a prevenção. $O$ tratamento dos resíduos é uma alternativa para destinar materiais contaminantes, fazendo com que eles necessitem de um manejo específico. Ela atua também promovendo competência a órgãos estaduais, municipais e ambientais quanto ao estabelecimento de critérios para licenciamento ambiental referente ao tratamento e destino dos RSS (BRASIL, 2006).

O PGRSS é um documento que descreve e aponta as ações referentes ao manejo dos resíduos, segregação, acondicionamento, identificação, coleta, armazenamento temporário, transporte, tratamento e disposição final. Este documento é um dos itens exigidos sanitariamente para as farmácias comunitárias receberem o licenciamento de saúde para funcionamento, estando indicado nas Boas Práticas Farmacêuticas para farmácia e drogarias pela RDC 44/2009 da Anvisa (BRASIL, 2009).

De acordo com Costa e Batista (2016), o gerenciamento dos RSS foi constituído juntamente com os procedimentos de implementação de bases científicas, gestão, normativas legais e entre outros, visto que, tinham como objetivo minimizar a produção de resíduos e proporcionar a eles destinação segura almejando proteção dos trabalhadores, da saúde pública e do meio ambiente.

Diante dos riscos ofertados ao meio ambiente e saúde pública, os resíduos sólidos foram classificados (segundo a NBR 10.004 de 2004) em duas classes: classe I e classe II. Os de classe I são considerados perigosos, os quais, possuem propriedades físicas, químicas ou biológicas possíveis de apresentarem riscos à saúde e ao meio ambiente, possuindo uma ou mais das seguintes propriedades: 
corrosividade, reatividade, inflamabilidade, toxicidade e patogenicidade. Já os de classe II não são considerados perigosos (BRASIL, 2006).

Segundo a RDC 222/2018 da Anvisa, os RSS são classificados em cinco agrupamentos de resíduos: do tipo $\mathrm{A}$ (Biológicos, com riscos de infecção), do tipo $\mathrm{B}$ (Substâncias químicas que têm a possibilidade de apresentar riscos à saúde pública ou do meio ambiente), do tipo $C$ (Materiais radioativos), do tipo $D$ (lixos sem riscos, comparados a resíduos domiciliares) e os do tipo $\mathrm{E}$ (Materiais perfurocortantes) (BRASIL, 2018).

Os RSS merecem atenção devido ao potencial contaminante e seu destino errôneo, que causa diversos impactos ambientais, levando a alterações na qualidade do solo, ar e corpo hídrico (DALBEN et al., 2015).

Assim, este estudo objetivou analisar a gestão dos resíduos sólidos declarados por farmácias comunitárias privadas, através dos PGRSS entregues à vigilância sanitária local como pré-requisito a obtenção do alvará sanitário do Município de Goiana, Pernambuco, um importante centro econômico equidistante das capitais pernambucana e paraibana, recém integrado a região metropolitana do Recife.

\section{METODOLOGIA}

O trabalho foi caracterizado como um estudo de prevalência, quantitativo e descritivo, sendo realizado no Município de Goiana, situado na Região Metropolitana do Recife, Pernambuco, durante os meses de junho a dezembro de 2019.

Para a pesquisa, foram usados como fonte de dados secundários, sem contato ou participação de humanos, os PGRSS que continham a Definição do Porte do Estabelecimento e Plano do Serviço, dos quais foram coletadas as informações cadastrais da empresa, o porte pela geração de RSS, as notas da responsabilidade técnica, dados da infraestrutura da farmácia, quantitativo de recursos humanos, estimativa de atendimentos na unidade de saúde e os tipos de resíduos gerados, assim como, as formas de acondicionamento e destino final. Os formulários de 
definição de porte e serviço entregues à vigilância municipal, são recebidos com aprovação da Agência Pernambucana de Vigilância Sanitária e disponibilizados online para preenchimento (CPRH, 2019).

Todas as informações foram obtidas na sede da vigilância local, em presença de funcionário, evitando o risco de vazamento de informações que comprometessem e indicassem as unidades avaliadas, ademais, o estudo obedeceu a Resolução $n^{\circ}$ 466/2012 do Conselho Nacional de Saúde e só foi desenvolvido após a aprovação da Direção da unidade de saúde.

\section{RESULTADOS E DISCUSSÃO}

A pesquisa foi realizada em 26 farmácias comunitárias situadas na sede do município e nos cinco distritos que, além de resíduos comuns, semelhantes ao lixo domiciliar, também produzem resíduos de materiais com potencial contaminante e perfurocortantes, devido aos serviços disponibilizados por algumas unidades.

A partir das planilhas da definição de porte, parte do PGRSS, é possível verificar as informações contidas na Tabela 1, sobre as médias de área construída, quantidade de funcionários e atendimentos, salientando que todas as farmácias se declararam com pequeno porte de estabelecimento de saúde, condizente com o encontrado Souza et al., 2019, onde houve predominância nas repostas das farmácias entre o pequeno e médio porte.

TABELA 1. INFORMAÇÕES REFERENTES DEFINIÇÃO DO PORTE DAS FARMÁCIAS DO LOCAL DE ESTUDO.

Variável Área construída $\left(\mathrm{m}^{2}\right)$

Número de funcionários

Número de serviços farmacêuticos por mês

Horas de funcionamento por dia

Quantidades de dias em funcionamento por semana
Média (mínimo - máximo)

$60,20(24,00-150,00)$

$05(01-11)$

$122,38(100-700)$

$12,17(09-16)$

$06(05-07)$

Fonte: Dados do estudo, 2019. *DP = Desvio padrão, 
De acordo com Melo et al. (2019), a evolução da medicina acresce em novas tecnologias para diagnósticos e tratamentos, com isso, cada vez mais materiais são gerados, os quais, podem proporcionar malefícios ao homem e ao meio ambiente. Seguindo a mesma linha de estudo, também foi pontuado por ele a expansão de uma farmácia estudada, no qual, foi possível identificar que quanto maior a proporção da mesma, maior é o número de atendimento ao cliente e consequentemente crescimento no número de profissionais (aproximadamente 39 empregos diretos), diferente do verificado no município desta pesquisa. Na Tabela 2 estão descritas as formas de acondicionamento e tratamento final destinados para cada tipo de resíduo declarado nos PGRSS entregues a vigilância sanitária municipal.

TABELA 2. TIPOS DE RESÍDUOS GERADOS PELAS 26 FARMÁCIAS DO ESTUDO, ACONDICIONAMENTO E TRATAMENTO INFORMADOS NOS PGRSS.

\begin{tabular}{ccccc}
\hline RSS & ACONDICIONAMENTO & TRATAMENTO & N & $\%$ \\
\hline A & $\begin{array}{c}\text { Bombonas de plástico rígido, com } \\
\text { saco indicativo de material biológico } \\
\text { Bombonas plásticas com sacos }\end{array}$ & Incineração & 05 & $19,23 \%$ \\
B & $\begin{array}{c}\text { Incineração } \\
\text { indicativos de material químico }\end{array}$ & 26 & $100 \%$ \\
C & Sacos plásticos para lixo comum & $\begin{array}{c}\text { Coleta municipal de } \\
\text { lixo - aterro sanitário }\end{array}$ & 26 & $100 \%$ \\
D & $\begin{array}{c}\text { Caixa para coleta de materiais } \\
\text { perfurocortantes }\end{array}$ & Incineração & 05 & $19,23 \%$ \\
\hline
\end{tabular}

Outro ponto abordado foi a quantidade de lixo gerado por cada farmácia, sendo declarado por todas as unidades que a produção de RSS estava na faixa de até $50 \mathrm{Kg} / \mathrm{mês}$, ainda, a média de lixo total gerado pelas farmácias foi de 03,09 $\mathrm{Kg} /$ mês (mínimo de 0,3 $\mathrm{Kg}$ e máximo de 15,00 Kg). Como apenas 05 farmácias disponibilizam serviços farmacêuticos de aferição da glicemia capilar, fonte geradora de resíduos dos tipos $A$ e $E$, os lixos comuns são responsáveis pela maior quantidade gerada em todas os estabelecimentos.

A RDC n 44/2009 (BRASIL, 2009), que estabelece os critérios e condições mínimas para o cumprimento das Boas Práticas Farmacêuticas para o controle sanitário do funcionamento, da dispensa, comercialização de produtos e prestação 
de serviços farmacêuticos em farmácias e drogarias, em seu artigo 93 menciona que: "é permitido farmácias e drogarias participar de programa de coleta de medicamentos a serem descartados pela comunidade, contribuindo assim com a coleta de medicamento descartados". Esta orientação dificilmente é cumprida e, pelo analisado nos PGRSS, não fica claro se as farmácias recebem medicamentos vencidos da população.

De acordo com Santos (2018) faz-se necessário o conhecimento quanto a classificação dos RSS, desta forma é possível melhoria e organização no gerenciamento dos resíduos, além da identificação dos mesmos a fim de observar os riscos as pessoas e ao meio ambiente. Pelos PGRSS avaliados, nas farmácias pesquisadas, os resíduos eram devidamente acondicionados e identificados para posterior tratamento.

Além disso, a destinação final dos resíduos é outro ponto importante, visto que, os locais devem estar preparados para o recebimento dos mesmos, sendo assim, Santos (2018) observou maior predominância de incineração na destinação dos RSS coletados, semelhante ao encontrado neste estudo e, também foi observado por Santos, que a destinação em aterro sanitário, muitas vezes realizada de forma indevida, faz com que os resíduos de saúde fiquem misturados com o lixo urbano.

$\mathrm{O}$ acondicionamento e descarte dos resíduos gerados devem estar de acordo com a RDC 222/2018 (BRASIL, 2018), ficando todas formas de acondicionamento e tratamentos finais respeitados pelas farmácias, com base nos PGRSS avaliados. Os agentes biológicos devem estar separados em sacos ou recipientes que evitem vazamento e que sejam resistentes a rupturas, sendo assim, é perceptível o cumprimento da norma, já que este tipo de lixo foi colocado em saco branco leitoso juntamente com bombonas de plástico rígido.

As substâncias químicas, que apresentam risco a saúde pública, devem ser acondicionadas em recipientes de material rígido e observando a compatibilidade química dos resíduos, se para finalizar o manejo destes RSS; os resíduos do grupo D não apresentam risco a saúde da população ou ao meio ambiente, os quais, podem ser comparados aos resíduos domiciliares, o que permite acondicionamento e tratamento finais mais simples que os demais. 
O grupo E deve obter caixas específicas para depósito de agulhas, ampolas de vidro e outros semelhantes, recebendo como tratamento a incineração, sendo assim, as farmácias pesquisadas respeitaram as normas outorgadas.

De acordo com Lustosa e Silva (2019), existem dados e comprovações quanto ao impasse causado pelo descarte inadequado dos resíduos de saúde e apesar de toda manifestação ainda falta conscientização da população em geral e até mesmo das gestões para que sejam observadas e realizadas boas práticas quanto a utilização e descarte corretos dos resíduos abordados.

Os riscos elencados pelos resíduos de saúde dependem também do grau de toxicidade e de quanto tempo os mesmos ficam em contato com o meio ambiente; esta concentração no meio ambiente (permanência dos produtos) podem agravar cada vez mais a degradação dos ecossistemas.

\section{CONCLUSÃO}

Após análise dos resultados foi possível constatar que as farmácias comunitárias participantes da pesquisa cumpriram com as imposições da legislação vigente. Sendo assim, as mesmas são responsáveis pelos resíduos gerados, bem como seu acondicionamento e tratamento.

Entretanto, apesar do cumprimento legislativo, faz-se necessária maior fiscalização quanto ao descarte dos resíduos de saúde, para sensibilização da população quanto aos danos causado ao meio ambiente e ao homem.

É perceptível que investimentos na educação em saúde devem ser realizados a fim de garantir cada vez mais recursos naturais para as gerações futuras.

\section{REFERÊNCIAS BIBLIOGRÁFICAS}

AGÊNCIA ESTADUAL DE MEIO AMBIENTE - CPRH. PRGSS com formulários. Governo do de Pernambuco: Recife, 2019. em: [http://www.cprh.pe.gov.br/Controle_Ambiental/licenciamento/tipologias/empreendimentos de_servicos_de_saude/pgrss/duvidas/41787\%3B69615\%3B480101160202\%3B0\%3B0.asp]. 
BRASIL. Agência Nacional de Vigilância Sanitária - ANVISA. RDC № 44 de 17 de agosto de 2009. Regulamenta as Boas Práticas Farmacêuticas e dá outras providências. Brasília: ANVISA, 2009.

BRASIL. Agência Nacional de Vigilância Sanitária. RDC N²22 de 28 de março de 2018. Regulamenta as Boas Práticas de Gerenciamento dos Resíduos de Serviços de Saúde e dá outras providências. Brasília: ANVISA, 2018.

BRASIL. Agencia Nacional de Vigilância Sanitária. RDC № 306, de 7 de dezembro de 2004. Dispõe sobre o Regulamento Técnico para o gerenciamento de resíduos de serviços de saúde. Brasília: Anvisa, 2004.

BRASIL. Casa Civil. Lei $n^{\circ}$ 12.305, de 02 de agosto de 2010. Institui a Política Nacional de Resíduos Sólidos; altera a Lei no 9.605, de 12 de fevereiro de 1998; e dá outras providências. Brasília: Presidência da República, 2010.

BRASIL. Ministério da Saúde. Agência Nacional de Vigilância Sanitária. Manual de gerenciamento de resíduos de serviços de saúde. Série A. Normas e Manuais Técnicos. 182p. Brasília: ANVISA, 2006.

COSTA, J. S. et al. Consciência ambiental em Humaitá: como ocorre o descarte dos resíduos sólidos nas empresas. Revista Educamazônia. v. 20, n. 1, p.55-62, 2018.

DERISIO, J. C. Introdução ao controle de poluição ambiental. 5ed. São Paulo: Oficina de Textos, 2017. 232p.

GONÇALVES, F. K.; FRANCO, Y. O.. O descarte de Medicamentos Vencidos e os Aspectos Toxicológicos da Incineração. Saúde Revista, Piracicaba. v.6, n.12, 2013.

LUSTOSA, J. H. N. C.; SILVA, M. M. Gerenciamento de resíduos farmacêuticos, medicamentos vencidos, no município de Corrente - Piauí (Brasil). Revista Brasileira de Meio Ambiente, v. 7 , n. 2, p.72-81, 2019.

MELO, N. O. et al. Estudo de caso- gerenciamento de resíduos em farmácias de manipulação de João Monlevade. Revista Observatorio de la Economía Latinoamericana. Jan, 2019.

PINHEIRO, L. A; SILVA, E. R. Estudos sobre resíduos sólidos de serviços de saúde e a educação ambiental. Revista Internacional de Ciências. v. 6, n. 1, p.21-28, 2016.

SANTOS, G. M. Tratamento e destinação final dos resíduos de serviços de saúde pública do hospital felipe jorge da cidade de São Bernardo do Estado do Maranhão. 2018. 46 f. TCC (Graduação) - Curso de Ciências Naturais/química, Universidade Federal do Maranhão, São $\quad$ Bernardo, $2018 . \quad$ Disponível em: <file:///C:/Users/Cliente\%20Preferencial/Downloads/Gest\%C3\%A3o\%20de\%20residuos.pdf>. Acesso em: 03 jan. 2020.

SANTOS, L. N. D.; RUIZ, J. B.; CAMILO, R. Proposta de gerenciamento de resíduos de saúde em unidades públicas do município de Umuarama, PR. Revista Tecnológica, v. 28, n. 1, p. 7484, 2017.

SOUZA, B. C. O. Q; PANASSOLLO, D. B.; PALMEIRA, D. C.; CAMPOS, M. M. N; SOUSA, M. S.; MAGALHÃES, R. C. Análise da gestão de resíduos medicamentosos em farmácias do Município De Santarém-PA. Revista Gestão e Sustentabilidade Ambiental. v. 8, n. 1, 2019. 